\title{
Comparison of magnetic field observations of an average magnetic cloud with a simple force free model: the importance of field compression and expansion
}

\author{
R. P. Lepping ${ }^{1}$, T. W. Narock ${ }^{1,2}$, and H. Chen ${ }^{3}$ \\ ${ }^{1}$ NASA, Goddard Space Flight Center, Heliophysics Science Division, Greenbelt, MD 20771, USA \\ ${ }^{2}$ Goddard Earth and Science Technology Center, University of Maryland, Baltimore County, MD, USA \\ ${ }^{3}$ River Hill High School, Clarksville MD 21029, USA
}

Received: 1 June 2007 - Revised: 6 November 2007 - Accepted: 14 November 2007 - Published: 2 January 2008

\begin{abstract}
We investigate the ability of the cylindrically symmetric force-free magnetic cloud (MC) fitting model of Lepping et al. (1990) to faithfully reproduce actual magnetic field observations by examining two quantities: (1) a difference angle, called $\beta$, i.e., the angle between the direction of the observed magnetic field $\left(B_{\text {obs }}\right)$ and the derived force free model field $\left(B_{\bmod }\right)$ and (2) the difference in magnitudes between the observed and modeled fields, i.e., $\Delta B\left(=\left|B_{\mathrm{obs}}\right|-\left|B_{\mathrm{mod}}\right|\right)$, and a normalized $\Delta B$ (i.e., $\Delta B /<B>$ ) is also examined, all for a judiciously chosen set of 50 WIND interplanetary MCs, based on quality considerations. These three quantities are developed as a percent of MC duration and averaged over this set of MCs to obtain average profiles. It is found that, although $\langle\Delta B\rangle$ and its normalize version are significantly enhanced (from a broad central average value) early in an average MC (and to a lesser extent also late in the MC), the angle $\langle\beta>$ is small (less than $8^{\circ}$ ) and approximately constant all throughout the MC. The field intensity enhancements are due mainly to interaction of the MC with the surrounding solar wind plasma causing field compression at front and rear. For example, for a typical MC, $\Delta B /<B>$ is: $0.21 \pm 0.27$ very early in the $\mathrm{MC},-0.11 \pm 0.10$ at the center (and $-0.085 \pm 0.12$ averaged over the full "central region," i.e., for $30 \%$ to $80 \%$ of duration), and $0.05 \pm 0.29$ very late in the MC, showing a double sign change as we travel from front to center to back, in the MC. When individual MCs are examined we find that over $80 \%$ of them possess field enhancements within several to many hours of the front boundary, but only about 30\% show such enhancements at their rear portions. The enhancement of the MC's front field is also due to MC expansion, but this is usually a lesser effect compared to compression. It is expected that this compression is manifested as significant distortion to the MC's crosssection from the ideal circle, first suggested by Crooker et
\end{abstract}

Correspondence to: R. P. Lepping

(ronald.p.lepping@nasa.gov) al. (1990), into a more elliptical/oval shape, as some global MC studies seem to confirm (e.g., Riley and Crooker, 2004) and apparently also as confirmed for local studies of MCs (e.g., Hidalgo et al., 2002; Nieves-Chinchilla et al., 2005).

Keywords. Solar physics, astrophysics and astronomy (Flares and mass ejections, Magnetic fields) - Space plasma physics (experimental and mathematical techniques)

\section{Introduction}

We are concerned here with evaluating the capability of the force-free cylindrically symmetric (and so-called constant alpha) magnetic cloud (MC) fitting model of Lepping et al. (1990) to reproduce average input data (average magnetic field vector observations), for relatively good quality MCs, as defined below. This model is based on MC properties earlier discussed by Burlaga et al. (1981), Goldstein (1983), and Burlaga (1988). A MC was defined empirically by L. Burlaga and coworkers as a (usually large) interplanetary structure having enhanced field magnitude, a relatively smooth change in field direction as the spacecraft passes through the MC, and lower proton temperature (and proton beta) than the surrounding solar wind (see, e.g., Burlaga, 1995). In particular, we analyze here the Lepping et al. (1990) model's average ability to provide separately field directions and field magnitudes that are consistent with observations using a large number of WIND MCs. It is well know that this model is relatively good at reproducing the actual direction of the field for most events (and it generally provides good estimates of most of the model fit parameters, especially axial attitude, Lepping et al., 2003a, 2004), but its ability to capture the field magnitude profile across the MC is almost always poor. This is believed to be mainly due to the two oversimplifying assumptions that were employed in the model: (1) circular cross-section and (2) static nature of

Published by Copernicus Publications on behalf of the European Geosciences Union. 
the MC (i.e., non-expanding). So we ask, "How faithful, on average, is the field directional-profile reproduction, and how poor is the field magnitude-profile reproduction by the model - and where is it poorest"?

Lepping et al. (2003a, also see 2004) took a different approach in studying the Lepping et al. (1990) MC fitting model's ability to reproduce observations by showing how the "fluctuation level" of the field within a MC can propagate to the seven MC fit-parameter error estimates; the higher the fluctuation level the higher the parameter uncertainty. (Errors due to choosing incorrect boundary times were not considered. ) Other independent variables affecting uncertainty, besides the fluctuation level, were the closest approach distance and the size of the oblique angle that the spacecraft's path makes with the MC's axis. Such error estimates for the MC fit-parameters hold for the given MC as a whole. The study here is concerned with finding quantitatively how field observations typically deviate from the MC model field, as a function of percent travel through the average $M C$, i.e., as a function of space, and with any implications.

\section{Analysis and results: field direction}

We start by defining a difference angle (called $\beta$ ), which is that angle between the direction of the observed magnetic field within a MC and that direction of the field derived by the Lepping et al. (1990) MC fitting model (assumed for the same instant front and rear. For examplind it convenient to present $\beta$ as a percent of MC duration through the MC, so we can superimpose many MCs of various actual sizes to find an "average" case. We, in fact, do this using a large number of good quality WIND MCs; quality $\left(Q_{O}\right)$ is defined in Lepping et al. (2006). That is, only MCs of $Q_{O}$ of 1 or 2 are used, i.e., those of $Q_{O}=3$, quantitatively determined poor cases, are ignored in forming the average $\beta$. These $Q_{O}=1,2$ cases comprise $50 \mathrm{MCs}$ out of an original 82 , for the interval from WIND launch until August 2003. We determined that it is very important to smooth the field observations, through filtering, before any difference quantity is formed; this smoothing is done with three trial running averages to be explained below. We justify this field-smoothing on the basis of our desire to judge the MC parameter fit-model's ability to track the major component of the observed MC, i.e., we do not wish to follow all the little wiggles in the field that are usually due to waves and/or discontinuities superimposed on the MC's field and which are not considered part of the MC (see, e.g., Narock and Lepping, 2007).

First, we will discuss how average $\beta(<\beta>)$ is derived. We start by "low pass filtering" the one minute averages of the magnetic field within each MC of the 50 cases of interest with a running average filter, in order to smooth the observations, as mentioned above. [The $1 \mathrm{~min}$ averages were derived by averaging WIND/MFI measurements that were made at the standard sample rate of $\approx 11$ vector sam- ples/s (usually) done in component form.] This is done for three trial smoothing-intervals (denoted $\Delta T$ ) of 15, 61, and $242 \mathrm{~min}$; see Lepping et al. (2007) for an identical analysis using five smoothing intervals. (No interval lengths longer than 242 min were attempted.) After this, the resulting MCs are still in one minute average form. We then divide the duration of the MC into 100 evenly spaced buckets, so that each bucket represents one percent of the duration of that MC. In this way we can superimpose the 50 MCs regardless of the actual differences in their durations. [Since we limit the study to only the better quality MCs, $Q_{O}$ of 1 and 2 cases (see Lepping et al., 2006), we are less likely to badly distort the results by those extreme cases of very distant passages where only a very small outer segment of the MC would be contributing to the average. Actually, only six cases of the 50 had closest approaches greater than $66 \%$ of the radius.] However, by employing percent-duration means that the original 1 min averages must be averaged in each MC separately to whatever interval is necessary to create the 100 averages across the MC. Typically such an average is about $13 \mathrm{~min}$, since the average MC-duration was $21.6 \mathrm{~h}$ for these $50 \mathrm{MCs}$ (e.g., see Lepping et al., 2006). The model field, however, was based on the discrete averages (which could have been any one of $\Delta T^{\prime}=15,30$, or 60 min depending on the duration of the MC); the results are shown in Table 2 of the Website: http://lepmfi.gsfc.nasa.gov/mfi/mag_cloud_S1.html

It is obvious that there was no need for "model-curve smoothing", because the resulting model-field is already represented as a smoothed vector field, in time, from the Lepping et al. (1990) fitting procedure itself. We pick 100 evenly spaced vectors from the model-field for each MC and, along with the 100 matched observed fields, as described above, we find $100 \beta$ 's using

$\beta=\cos ^{-1}\left(\boldsymbol{B}_{\text {obs }} \cdot \boldsymbol{B}_{\text {mod }} /\left(\left|\boldsymbol{B}_{\text {obs }}\right|\left|\boldsymbol{B}_{\bmod }\right|\right)\right)$,

at each of the 100 points for each MC, where $\boldsymbol{B}_{\text {obs }}$ is the observed field and $\boldsymbol{B}_{\text {mod }}$ is the model field.

After these operations, the 100 points ( $\beta$ 's) of each of the $50 \mathrm{MCs}$ are averaged, and these averages and their associated standard deviations ( $\sigma$, i.e., the RMSs) are retained; the results are shown in Fig. 1, where the curves are labeled (a) to (c), according to the scheme of Table 1 . The central curves are the averages of $\beta$, and the length (top-to-bottom) of the vertical bar at each point represent twice the sigma for that point. It is obvious that set (a) is more jagged generally, has higher sigmas, and its average is higher than those of all the other curves. But what all three average curves have in common is an approximate constancy across the full $100 \%$ duration, except for some boundary influences. As might be expected, set (c), where the smoothing interval is largest at $242 \mathrm{~min}$, has the broadest boundary influence, but this set is the smoothest, has the lowest overall average value (of $7.6^{\circ}$, if the average is taken between $10 \%$ and $90 \%$ duration), and is the most constant between $10 \%$ and $90 \%$-duration. (The 
Table 1. The final averages and sigmas for $\beta^{*}, \Delta B^{+}$, and $\Delta B /<B>^{+}$for three smoothing interval lengths.

\begin{tabular}{|c|c|c|c|c|c|c|c|}
\hline Case & $\begin{array}{l}\text { Interval length } \\
\Delta T \text { (minutes) }\end{array}$ & $\begin{array}{c}<<\beta>> \\
(\text { Deg) }\end{array}$ & $\begin{array}{l}<\sigma_{\beta}> \\
(\mathrm{Deg})\end{array}$ & $\begin{array}{c}<<\Delta B>> \\
(\mathrm{nT})\end{array}$ & $\begin{array}{c}<\sigma_{\Delta B}> \\
(\mathrm{nT})\end{array}$ & $<<\Delta B /<B>>>$ & $<\sigma_{\Delta B /<B>}>$ \\
\hline (a) & 15 & 10.8 & 7.3 & -0.88 & 2.04 & -0.060 & 0.13 \\
\hline (b) & 61 & 9.7 & 6.4 & -0.93 & 2.06 & -0.064 & 0.14 \\
\hline (c) & 242 & 7.6 & 4.9 & -1.18 & 1.90 & -0.085 & 0.12 \\
\hline
\end{tabular}

* Average and sigma derived over the interval of $10 \%$ to $90 \%$ of duration for $\beta$.

+ Average and sigma derived over the interval of $30 \%$ to $80 \%$ of duration for $\Delta B$ and $\Delta B /<B>$.

Table 2. Comparisons of $<<\Delta B>>$ and $<<\Delta B /<B>>>$ within the Average Magnetic Cloud for the $\Delta T=242$ Minute Case.

\begin{tabular}{lcccc}
\hline \multicolumn{1}{c}{ Quantity Considered: } & $\begin{array}{c}<\Delta B>> \\
(\mathrm{nT})\end{array}$ & $\begin{array}{c}<\sigma_{\Delta B}> \\
(\mathrm{nT})\end{array}$ & $<<\Delta B /<B>>>$ & $<\sigma_{\Delta B /<B>>}$ \\
\hline Very early in the MC & 3.5 & 4.5 & 0.21 & 0.27 \\
At the Center & -1.6 & 1.8 & -0.11 & 0.10 \\
For the full "central region* & -1.2 & 1.9 & -0.085 & 0.12 \\
Very late in the MC & 0.60 & 3.4 & 0.05 & 0.29 \\
\hline
\end{tabular}

* This region refers to $30 \%$ to $80 \%$ of duration within the average MC, the "average-middle region" for study of field magnitude differences.

fact that duration of the enhanced boundary effect for $\beta$ depends on the filter-length, being almost negligible for the 15 min case, clearly indicates that these small variations in $\beta$ near the boundaries are due to filtering, are not real, and can be ignored.) The left side of Table 1 shows the averages and sigmas for $\beta$ (i.e., $<<\beta>>,<\sigma_{\beta}>$ ) derived from each of the three curves, over the interval of $10 \%$ to $90 \%$ to avoid boundary concerns. The $242 \mathrm{~min}$ average curve (c) gives the smallest $\left\langle<\beta>>\right.$ and $\left\langle\sigma_{\beta}>\left(4.9^{\circ}\right)\right.$, as mentioned above, and we will consider it to give the best estimate of $\langle<\beta>>$. Further we will assume that $\langle\beta>$ is approximately constant all across the average MC, because of the argument above concerning the $15 \mathrm{~min}$ case. The main point here is that for a fixed $\Delta T,\left\langle\beta>\right.$ and $\sigma_{\beta}$ are almost constant and small all across the average $\mathrm{MC}$, including the regions close to the boundary.

\section{Analysis and results: field magnitude}

We now examine the difference between the magnitudes of the observed (obs) and modeled (mod) fields $\left(\Delta B=\left|\mathrm{B}_{\mathrm{obs}}\right|-\left|B_{\mathrm{mod}}\right|\right)$, for the same time intervals as were studied for $\beta$, and also as a percent of MC duration through the MC, again for only $Q_{O}=1$ and 2 cases. These field intensity differences are processed in the same manner as $\beta$ with respect to smoothing and averaging, and the results are given in Fig. 2, which shows $\Delta B$ vs. \%-duration for the same three $\Delta T \mathrm{~s} 15$ to $242 \mathrm{~min}$ [i.e., (a) to (c)]. But we see that $\Delta B$ has very different characteristics compared to $\beta$. Whereas the three average $\beta$-curves of Fig. 1 are approximately sym- metric around $50 \%$ (and almost constant vs. \%-duration), the three $\Delta B$-curves are clearly not constant anywhere, not symmetric, and have roughly a broad "U" shape. The values of $<\Delta B>$ at the beginning of each panel are relatively high and the associated sigmas are also high. The middle columns of Table 1 show the averages and sigmas for $\Delta \mathrm{B}(<<\Delta B>>$, $<\sigma_{\Delta B}>$ ) derived from each of the three curves in Fig. 2 over the interval of $30 \%$ to $80 \%$ of duration, where the curves are near minimum, mostly negative, and deviate only slightly from being flat. [In a report by Lepping et al. (2007) where $|\Delta B|$ is examined (as well as $\Delta B$ itself), it is seen that in the region from $30 \%$ to $80 \%$ of duration of the average $\mathrm{MC}$ the ordinate value is relatively steady much like $\beta$ is in the region of $10 \%$ and $90 \%$-duration; see Figs. 7, 8 and 9 of that report. Hence, the region between $30 \%$ and $80 \%$ of duration is separated from the two "boundary" regions $(<30 \%$ and $>80 \%$ ), as in Figs. 2 and 3 and in Table 1 here. But $|\Delta B|$ itself will not be discussed here.] As Table 1 shows, the changes in these quantities vs. $\Delta T$-length (a to c) is also very regular, with the $\Delta T=242 \mathrm{~min}$ (c) giving the smallest average sigma of $\left\langle\sigma_{\Delta B}>\right.$ of $1.9 \mathrm{nT}$, yielding a $<<\Delta B>>$ of $-1.2 \mathrm{nT}$. However, it is interesting that $<<\Delta B>>$ and $<\sigma_{\Delta B}>$ are almost independent of the value of $\Delta T$, especially so for $\left\langle\sigma_{\Delta B}\right\rangle$. The $\Delta B$ results are clearly less sensitive to $\Delta T$ than the $\beta$ results.

Since the average fields across these 50 MCs cover a very broad range (from $5 \mathrm{nT}$ to $31 \mathrm{nT}$ ), and with model-estimated axial values covering the range of $9 \mathrm{nT}$ to $47 \mathrm{nT}$ (e.g., see Lepping et al., 2006), it is often reasonable and informative to normalize each MC's $\Delta B$ by its average $<B>$. Then all 


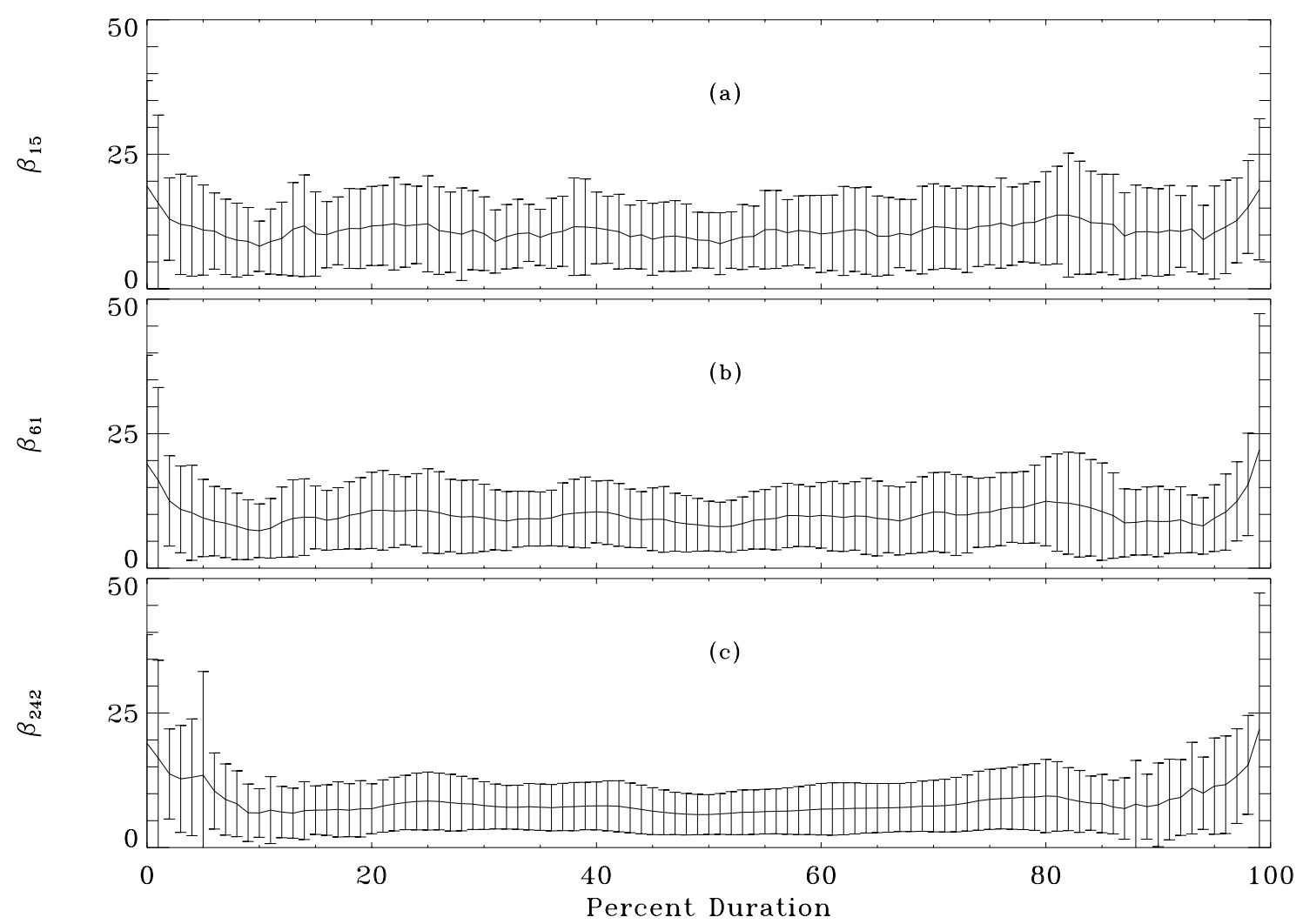

Fig. 1. Family of plots of $\beta$ vs. $\%$-duration for three $\Delta \mathrm{T}$ s (from top to bottom: 15,61 , and 242 min, as shown by the subscripts on the $\beta$ 's on the left). The central curves are the averages over the $50 \mathrm{MCs}$, and the lengths (top-to-bottom) of the vertical bars at the points represent twice their sigmas.

of those in the full set of 50 MCs are on the same footing when they are superimposed, when finding the overall average MC. In fact, superimposing such a normalized quantity probably makes more sense than simply superimposing $\Delta B$ itself. Therefore, in Fig. 3 we show the normalized $\Delta B$ (i.e., $\Delta B /<B>$ ) vs. \%-duration for the same three $\Delta T \mathrm{~s}$. The right side of Table 1 shows $<<\Delta B /<B>>>$ and $<\sigma_{\Delta B / B}>$ derived from each of the three curves in Fig. 3, again over the region of $30 \%$ to $80 \%$ of duration, and where again the regions near the boundaries are ignored. It is interesting that in this "middle" region the model gives a slightly higher estimate than the observations, (i.e., $<\langle\Delta B\rangle>$ and $<\langle\Delta|<B>>>$ are both negative), as Table 1 shows, unlike the early or later regions. Table 1 also shows that, for this central region, there is little variation of either $<\langle\Delta /<B>>>$ or its sigma as $\Delta T$-length is changed over 15 to $242 \mathrm{~min}$, as was true for $\langle\Delta B>$ itself, with however $<\sigma_{\Delta B / B}>$ being the smallest (0.12) for $\Delta T$ of $242 \mathrm{~min}$, where $<<\Delta B /<B>>>=-0.085$. We also notice that for $\Delta T$ of $15 \min |<<\Delta \mathrm{B} /<\mathrm{B}>>>|$ is the smallest, 0.060. The boundary regions obviously would show larger values of both $|\Delta B|$ and its $<\sigma_{\Delta B}>$.
As Figs. 2 an 3 show the model does not do so well in estimating field intensity closer to the boundaries in terms of either $\langle\Delta B>$ or $\langle\Delta B /<B>>$. In particular, for a "typical," good MC (i.e., for $Q_{O}=1,2$ quality cases where the smoothing average is $242 \mathrm{~min}), \Delta B$ is about $3.5 \mathrm{nT} \pm 4.5 \mathrm{nT}$ very early in the MC, $-1.6 \mathrm{nT} \pm 1.8 \mathrm{nT}$ in the middle (which for the full region of $30 \%$ to $80 \%$ of duration is $-1.2 \mathrm{nT} \pm 1.9 \mathrm{nT}$; see Table 1), and $0.60 \mathrm{nT} \pm 3.4 \mathrm{nT}$ at the end. We consider the $242 \mathrm{~min}$ case here, because it gives the lowest $\left\langle\sigma_{\Delta B}\right\rangle$ $(=1.9 \mathrm{nT})$ in that middle range of durations, as Table 1 shows. See Table 2 for a comparison of $\Delta \mathrm{B}$ (and $\sigma_{\Delta B}$ ) at various key locations in the average MC. Similarly for such a typical MC, $\Delta B /<B>$ is $0.21 \pm 0.27$ very early in the MC, about $-0.11 \pm 0.10$ at the center (and for the full region of $30 \%$ to $80 \%$ of duration is $-0.085 \pm 0.12$; see Table 1$)$, and $0.05 \pm 0.29$ late in the MC, as Fig. 3 and Table 2 show. So the quantities $|<\Delta B>|$ and $|<\Delta B /<B>>|$ very near the front are larger than the average-middle region by factors of 2.2 and 1.6, respectively. We also see that there is a sign change of $\langle\Delta B>$ (and $\langle\Delta B /<B>>$ ) from either of the near-boundary values (being positive) to the central values, which are negative. Obviously any individual MC can have difference-values that are quite at variance from these 


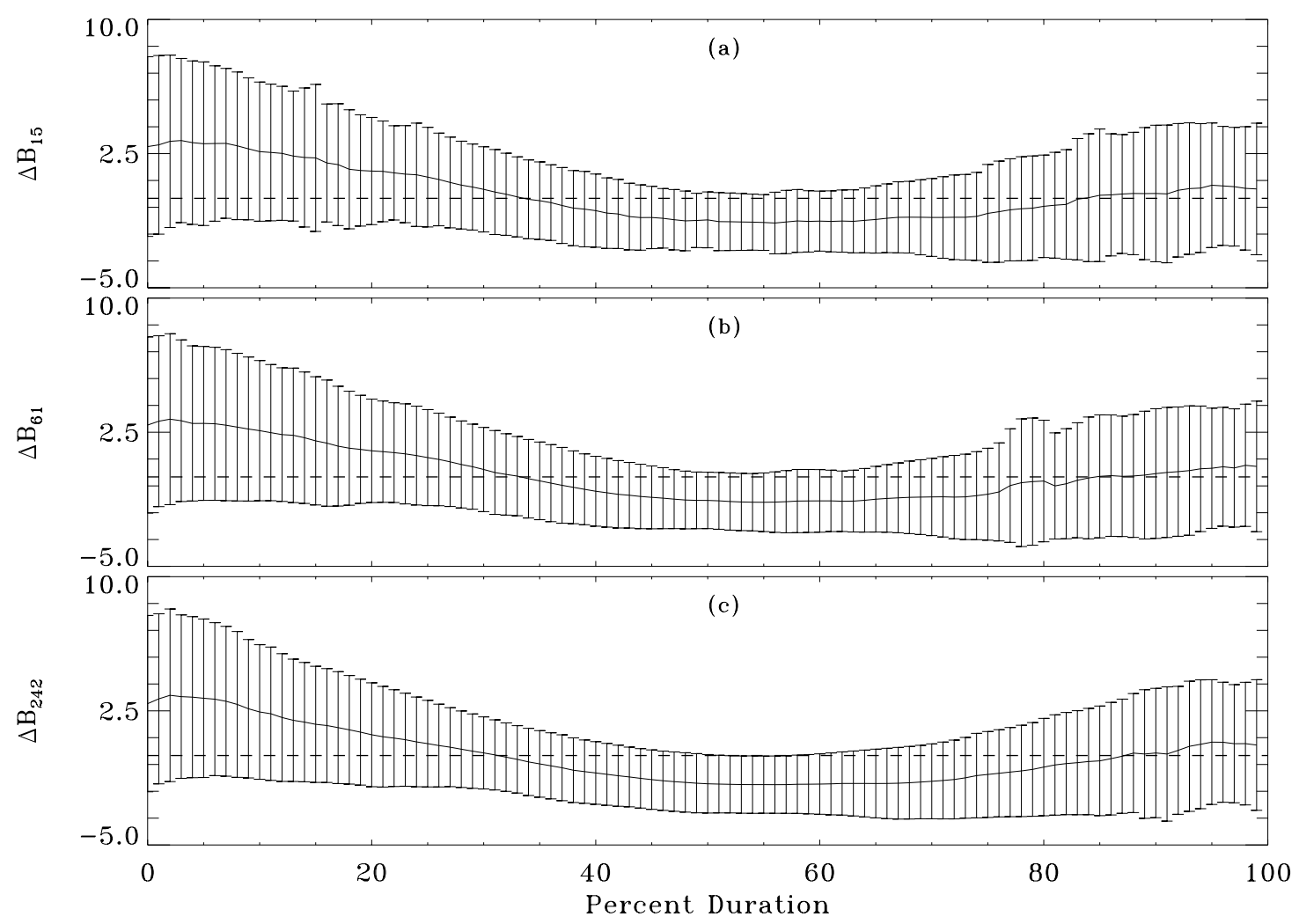

Fig. 2. Family of plots of $\Delta B\left(=B_{\mathrm{obs}}-B_{\text {mod }}\right)$ vs. \%-duration for three $\Delta T$ s (from top to bottom as in Fig. 1a, b, c, as shown by the subscripts on the $\Delta \mathrm{Bs}$ on the left). The central curves are the actual averages over the $50 \mathrm{MCs}$, and the lengths (top-to-bottom) of the vertical bars at the points represent twice their sigmas. The dashed line is the zero-line.

average values, for $\Delta B$, or $\Delta B /<B>$, as indicated by the uncertainty bars in Figs. 2 and 3.

The enhancements of field magnitude at the front and rear of a MC over the model predicted field are probably due mainly to the interaction of the MC with the surrounding solar wind plasma, compressing the MC and its field lines at the front and sometimes (as we discuss below) in the rear. The enhancement of the front's field is also due, to some extent, to MC expansion (see Klein and Burlaga, 1982; Burlaga and Behannon, 1982; and Lepping et al., 2003b).

Finally, we point out that an even larger set of WIND MCs $(N=100$, taken from launch until 13 April of 2006) were examined individually, in order to see if $\Delta B$ was $>0,<0$, or $\approx 0$ in the early few hours ("the front") of the MC and in the last few hours ("the rear"), separately. For the front, it was determined that about $81 \%$ of the cases showed $\Delta B>0$ (i.e., $B_{\text {obs }}>B_{\text {mod }}$ ), and the rest were mostly $\Delta B \approx 0$, with only $6 \%$ being $\Delta B<0$ (i.e., $B_{\bmod }>B_{\text {obs }}$ ); two of the $N=100$ were ambiguous and therefore not counted. For the rear, the three possibilities were distributed very differently: each was $33 \%$ (one of the $N=100$ was ambiguous and not counted). So apparently compression at the rear of a MC occurs less frequently than at the front by a factor of 2.3 . But when rear compression does occur, it appears stronger than at the front and is caused principally by impinging solar wind streams, or on occasion by impinging interplanetary shock waves. It is the impinging shocks at the rear that tilt the strength of the compression in favor of the rear, even though it is much less frequent. By contrast, front compression is caused by the MC overtaking the upstream solar wind usually causing an upstream shock depending on physical conditions upstream.

\section{Summary and discussion}

This analysis of 50 WIND MCs has shown that the Lepping et al. (1990) MC parameter fitting model is capable of obtaining relatively small directional differences between smoothed observational fields and the model fields, viz., angles to within about $\Delta \beta=8^{\circ}$, everywhere across an average MC for relatively good cases of individual MCs. [Results of three smoothing interval lengths $(\Delta T)$ were examined: 15,61 , and 242 minu. None of our results was strongly dependent on the choice of smoothing interval length, but we choose those based on $\Delta T=242 \mathrm{~min}$ as being marginally the best.] But field intensity differences vary quite markedly as a percent 


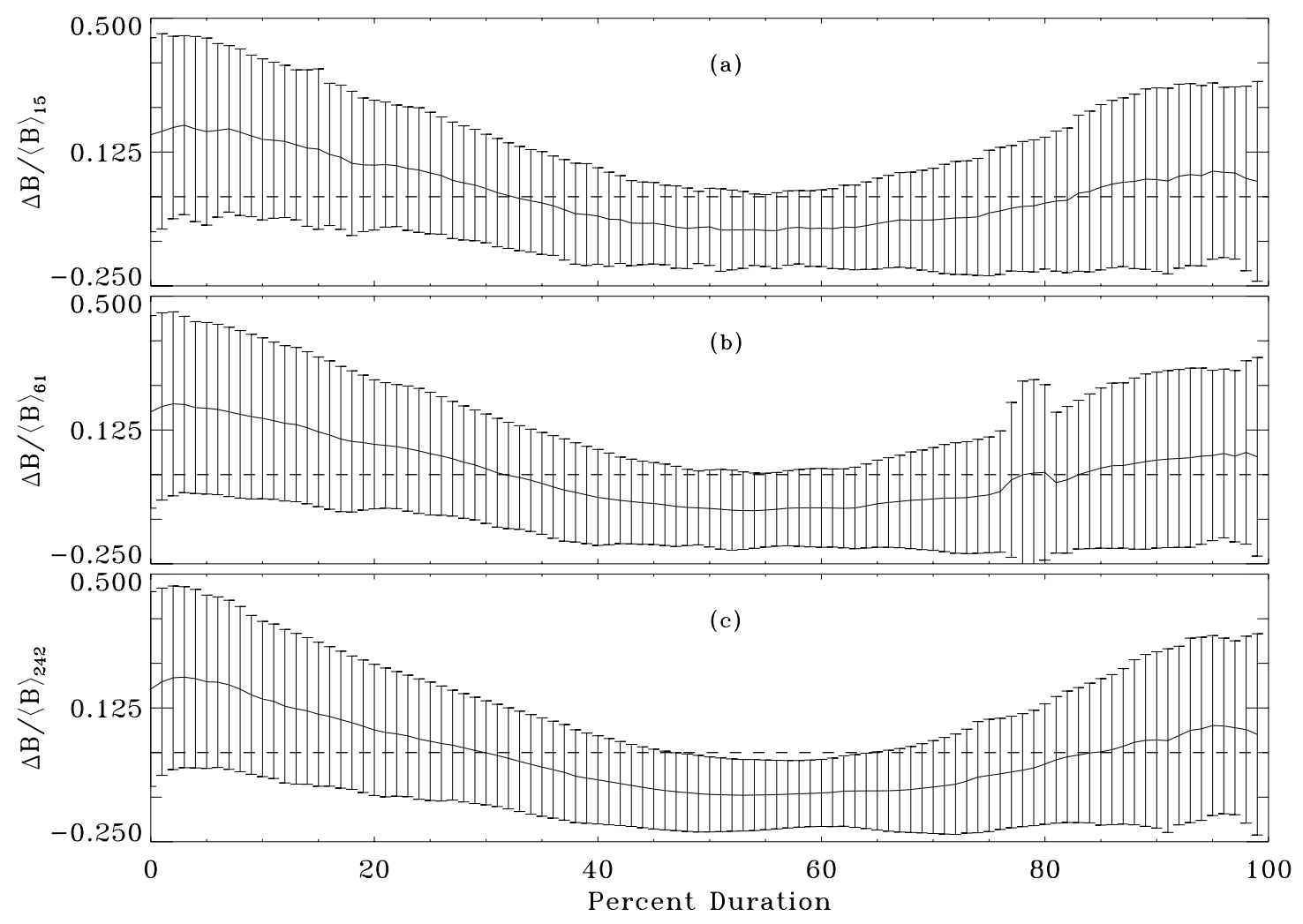

Fig. 3. Family of plots of $\Delta B /<B>$ (i.e., $\Delta B$ normalized by average B-magnitude) vs. \%-duration for three $\Delta T$ s (from top to bottom as in Fig. 1a, b, c), as shown by the subscripts on the $(\Delta B /<B>)$ s on the left). The central curves are the actual averages over the $50 \mathrm{MCs}$, and the lengths (top-to-bottom) of the vertical bars at the points represent twice their sigmas. The dashed line is the zero-line.

of travel through the MC, as measured by both $\langle\Delta B>$ and $<\Delta B /<B>>$. These quantities, especially near the average MC boundaries, i.e., earlier than $30 \%$ or later than $80 \%$ duration, are significantly larger than in the middle. At the front this increase is by a factor of 2.2 for $|<\Delta B>|$ and a factor of 1.9 for $\mid\langle\Delta B|<B>>|$. Specifically for $\Delta B|<B>$, in a typical MC, $\Delta B /<B>$ is: $0.21 \pm 0.27$ very early in the $\mathrm{MC},-0.11 \pm 0.10$ at the center, $-0.085 \pm 0.12$ averaged over the full "central region" (i.e., $30 \%$ to $80 \%$ of duration), and $0.050 \pm 0.29$ very late in the MC. Note that $B_{\mathrm{obs}}>B_{\text {mod }}$ in the front, $B_{\text {obs }}<B_{\text {mod }}$ in the middle, and back to $B_{\text {obs }}>B_{\text {mod }}$ near the rear boundary. The increase of the observed field magnitude over the model field near the boundaries is apparently principally indicative of field compression, but not uniquely. Other important effects (listed below) may contribute to this violation of the cylindrically symmetric force free model. We point out that all three quantities, $\beta, \Delta B$, and $\Delta B /<B>$ are independent of coordinate system being used for the study.

It is well know that interaction of a MC with the surrounding solar wind can cause field compression (e.g., Burlaga, 1995; Riley and Crooker, 2004). The question remains as to why the consequences of such an interaction are detected in $\Delta B$, but essentially not in $\beta$, within the $\mathrm{MC}$, at least not at $1 \mathrm{AU}$. It seems obvious why the field magnitude increases in the front and rear in response to compression, especially since the fields at the boundaries, being approximately aligned with the boundaries, are usually close to normal to solar wind flow (see Lepping and Berdichevsky, 2000), or at least have a significant component that is. (This will not be true for the unusual cases that represent observations at the extreme flanks of the global MC.) But the lack of response by the field's direction to compression is less obvious. Apparently it is due to the other side of the same argument: a MC's field, which is usually (and ideally in the model) aligned with the front and rear boundaries at and near these boundaries, is not expected to change direction significantly when these boundaries are compressed. Any indication of field compression via field directional change will not occur until the spacecraft gets well into the MC where the effect should be far less noticeable.

MCs from a larger data set of $N=100$ WIND cases (covering launch to mid April 2006) were examined individually to see how often front or rear MC compression occurred. (For this study it was assumed that $B_{\text {obs }}>B_{\text {model }}$ for several hours near the boundary indicated compression.) For the front it was determined that in $81 \%$ of the cases 
compression occurred. For the rear it occurred for only $33 \%$ of the cases. But when rear compression did occur, it was typically stronger than what was generally seen at the front.

MC expansion is also expected to play a role in $\Delta B$ distortion as a function of percent-duration of travel through a MC (see, e.g., Farrugia et al., 1992; Osherovich et al., 1993a, b; Berdichevsky et al., 2003), but it is expected to be secondary to compression (e.g., Burlaga, 1995; Riley and Crooker, 2004) and be less noticeable. And MC expansion should also cause asymmetry in the B-profile, causing the peak in $|\mathrm{B}|$ to move to an earlier time (from the center ideally) in a somewhat predictable way, depending on the gradient in solar wind speed across the MC (see, e.g., Farrugia et al., 1992 and Lepping et al., 2003b). As shown by Riley and Crooker (2004) using a global MHD model of MC evolution from the Sun, and allowing both spherical expansion and uniform expansion due to pressure gradients between the ejecta and the ambient solar wind, the cross-section of the MC as it moves outward takes on an elongated shape where ideally the long axis is approximately perpendicular to the Ecliptic plane, and where at $1 \mathrm{AU}$ its "ellipticity" is quite large; see Fig. 4 of Riley and Crooker (2004). This effect (related to the pressure gradient) is essentially what we suggest is equivalent to a measure of the compression of the $\mathrm{MC}$, in the sense that the ratio of the long- to the short-axis of the cross-section is, in some respect (depending on such details as the inclination of the MC's axis with respect to the Sun-spacecraft line and gradient of speed across the MC responsible for the expansion), a measure of this effect. Crooker et al. (1990) were the first to suggest that the cross-sections of some MCs may be significantly distorted from a circle, and support for this idea was given by Lepping et al. (1998), who showed from statistical analysis of many WIND MCs that there was a tendency for the long axis of the average ellipse to be normal to the Ecliptic plane, consistent with the findings of Riley and Crooker (2004), except that these later findings indicated that the effect is much more severe than originally thought.

It would be interesting to see the results of alternative MC models analyzed in the same manner as we studied the Lepping et al. (1990) model here and as applied to the same data set, if possible. Some of these alternatives include flux rope models employed by Farrugia et al. (1992) (expanding forcefree of constant alpha), Farrugia et al. (1999) (uniform twist of field in MC), Marubashi $(1986,1997)$ (force free with effect of solar rotation), Vandas et al., 2002 (3-D MHD simulations of MC compression), Hidalgo et al. (2002) (nonforce free approach to the topology of MCs), Vandas and Romashets (2003) (an oblate cylinder generalization of the Lundquist (1950) solution), Nieves-Chinchilla et al. (2005) (examples of MCs with non-circular cross-section and expansion), Vandas et al. (2006) (cross-sectional oblateness and expansion), and Marubashi and Lepping (2007) (torusshaped MCs, especially for long duration cases); see Riley et al. (2004) for comments on these and references to other MC parameter-fitting techniques. These models and stud- ies generally represent marked improvements over the simple force free flux rope of circular cross-section and might be expected to yield more satisfying results, especially with regard to the field magnitude profile of the $M C$, which means that the magnitude-differences should be small and uniform across the full average MC for a good model, just as was the case for $\beta$ in the Lepping et al. (1990) model.

The motivation for this present study started with a desire to make the most effective modifications to the Lepping et al. (1990) model, and it was believed that such a magnetic field difference-analysis, as presented here, should help guide us on what factors (and in what order) to concentrate on for such improvements. It is clear that accounting for MC compression (causing non-circular cross-section of the flux rope) will be one of the most important factors to consider. The already known successes of the above referenced studies by other teams, especially with regard to MC expansion and flux rope curvature for some cases should also be other key guides for improvement, as well as for the already attempted approaches (also given above) on non-circular cross-section flux rope accommodation. Probably the use of the torus geometry (or something similar) is best for analyzing MCs of long duration, as observed on the flanks of the global MC (Marubashi and Lepping, 2007), to account for flux rope curvature, as the authors effectively argue.

Acknowledgements. We thank A. Szabo and F. Mariani for WIND/MFI data handling and calibration, and are grateful to C.C. Wu and D. Berdichevsky for helpful comments and general assistance over the last few years on this and related magnetic cloud studies and to Charles Farrugia for comments on magnetic cloud compression. This work was supported by the NASA Heliophysics Guest Investigator Program.

Topical Editor B. Forsyth thanks two anonymous referees for their help in evaluating this paper.

\section{References}

Berdichevsky, D. B., Lepping, R. P., and Farrugia, C. J.: On Geometric Considerations of the Evolution of Magnetic Flux Ropes, Phys. Rev. E, 67(3), id. 036405, 2003.

Burlaga, L. F.: Magnetic clouds: Constant alpha force-free configurations, J. Geophys. Res., 93, 7217-7224, 1988.

Burlaga, L. F.: Interplanetary Magnetohydrodynamics, Oxford Univ. Press, New York, 89-114, 1995.

Burlaga, L. F., Sittler Jr., E. C., Mariani, F., and Schwenn, R.: Magnetic loop behind an inter- planetary shock: Voyager, Helios, and IMP-8 observations, J. Geophys. Res., 86, 6673-6684, 1981.

Burlaga L. F. and Behannon, K. W.: Magnetic clouds: Voyager observations between 2 and 4 AU, Solar Phys., 81, 181-192, 1982.

Crooker, N. U., Gosling, J. T., Smith, E. J., and Russell, C. T.: A bubble-like coronal mass ejection flux rope in the solar wind, in: Physics of Magnetic Flux Ropes, edited by: Russell, C. T., Priest, E. R., and Lee, L. C., 365-372, AGU GM 58, American Geophysical Union, Wash. D.C., 1990.

Farrugia, C. J., Burlaga, L. F., Osherovich V., and Lepping, R. P.: A comparative study of expanding force-free constant alpha mag- 
netic configurations with application to magnetic clouds, in Solar Wind Seven, edited by: Schwenn, T., p. 611, Pergamon New York, 1992.

Farrugia, C. J., Janoo, L., Torbert, R. B., Quinn, J. M., Ogilvie, K. W., Lepping, R. P. Fitzenreiter, R. J., et al.: Uniform-twist magnetic flux tube in the solar wind, Solar WIND 9, p. 745, edited by: Habbal, S., Esser, R., Hollweg, J. and Isenberg, P., 1999.

Goldstein, H.: On the field configuration in magnetic clouds, in Solar Wind Five, edited by: Neugebauer, M., NASA Conf. Publ., 2280, 731-733, 1983.

Hidalgo, M. A., Cid, C., Vinas, A. F, and Sequeiros J.: A non-force free approach to the topology of magnetic clouds in the solar wind, J. Geophys. Res., 107(A1), 1002, doi:10.1029/2001JA900100, 2002.

Klein L. W. and Burlaga, L. F.: Interplanetary magnetic clouds at 1 AU, J. Geophys. Res., 87, 613-624, 1982.

Lepping, R. P., Jones, J. A., and Burlaga, L. F.: Magnetic field structure of interplanetary magnetic clouds at $1 \mathrm{AU}, \mathrm{J}$. Geophys. Res., 95, 11 957-11 965, 1990.

Lepping, R. P., Berdichevsky, D., Szabo, A., Goodman, M., and Jones, J.: Modifications of magnetic cloud model: Elliptical cross-section, AGU EOS Transactions (SH11A-05), 79, F696, 1998.

Lepping, R. P. and Berdichevsky, D.: Interplanetary magnetic clouds: Sources, properties, modeling, and geomagnetic relationship, Research Signpost, Recent Res. Devel. Geophysics, 3, 77-96, 2000.

Lepping, R. P., Berdichevsky, D., and Ferguson, T.: Estimated errors in magnetic cloud model fit-parameters with force free cylindrically symmetric assumptions, J. Geophys. Res., 108(A10), 1356, doi:10.1029/2002JA009657, 2003a.

Lepping, R. P., Berdichevsky, D., Szabo, A., Arqueros, C., and Lazarus, A. J.: Profile of an average magnetic cloud at $1 \mathrm{AU}$ for the quiet solar phase: WIND Observations, Solar Phys., 212, 425-444, 2003b.

Lepping, R. P., Berdichevsky, D, and Ferguson, T: Correction (to paper entitled Estimated errors in magnetic cloud model fit-parameters with force free cylindrically symmetric assumptions by the same authors), J. Geophys. Res., 109, A07101, doi10.1029/2004JA010517, 2004.

Lepping, R. P., Berdichevsky, D. B., Wu, C.-C., A. Szabo, A., Narock, T, Mariani, F, Lazarus, A. J., and Quivers, A. J.: A summary of WIND magnetic clouds for the years 1995-2003: Model-fitted parameters, associated errors, and classifications, Ann. Geophys., 24(1), 215-245, 2006.
Lepping, R. P., Narock, T. W., and Chen, Henry: Unique vector features of the magnetic fields of interplanetary magnetic clouds as cylindrically symmetric force-free flux ropes, NASA/GSFC, Heliophysics Science Division, Internal Report, May 2007.

Lundquist, S.: Magnetohydrostatic fields, Ark. Fys., 2, 361, 1950.

Marubashi, K.: Structure of the interplanetary magnetic clouds and their solar origins, Adv. Space Res., 6(6), 335-338, 1986.

Marubashi, K.: Interplanetary magnetic flux ropes and solar filaments, In: Coronal Mass Ejections, Geophys. Monogr. Ser., Vol. 99, edited by: Crooker, N., Joselyn, J., and Feynman, J., 147156, AGU, Washington D. C., 1997.

Marubashi, K. and Lepping, R. P.: Long-duration magnetic clouds: A comparison of analyses using torus- and cylindrical-shaped flux rope models, Ann. Geophys., in press, 2007.

Narock, T. W. and Lepping, R. P.: Anisotropy of magnetic field fluctuations in an average interplanetary magnetic cloud at $1 \mathrm{AU}$, J. Geophys. Res., 112(A6), A06106, doi:10.1029/2006JA11987, 2007.

Nieves-Chinchilla, T., Hidalgo, M. A., and Sequeiros, J.: Magnetic clouds observed at $1 \mathrm{AU}$ during the period 2000-2003, Solar Phys., 232, 105-126, 2005.

Osherovich, V. A., Farrugia, C. J, and Burlaga, L. F.: Non-linear evolution of magnetic flux ropes: 1 . The low beta limit, J. Geophys. Res., 98, 13 225-13231, 1993a.

Osherovich, V. A., Farrugia, C. J., and Burlaga, L. F.: Dynamics of aging magnetic clouds, Adv. Space Res., 13(6), 57-62, 1993 b.

Riley, P. and Crooker, N. U.: Kinematic treatment of coronal mass ejection evolution in the solar wind, Ap. J., 600, 1035-1042, 2004.

Riley, P., Linker, J. A., Lionello, R., Mikic, Z., Odstrcil, D., Hidalgo, M. A., Cid, C, Hu, Q., Lepping, R. P., Lynch, B. J., and Rees, A.: Fitting flux ropes to a global MHD solution: A comparison of techniques, J. Atmos. Sol.-Terr. Phy., 66(15-16), 13211331, 2004.

Vandas, M., Odstrcil, D., and Watari, S.: Three-dimensional MHD simulation of a loop-like magnetic cloud in the solar wind, J. Geophys. Res., 107(A9), 1236, doi:10.1029/2001JA005068, 2002.

Vandas, M. and Romashets, E. P.: Force-free field with constant alpha in an oblate cylinder: A generalization of the Lundquist solution, Astron. Astrophys., 398, 801-807, 2003.

Vandas, M., Romashets, E. P., Watari, S., Geranios, A., Antoniadou, E., and Zacharopoulou, O.: Comparison of force-free flux rope models with observations of magnetic clouds, Adv. Space Res., 38, 441-445, 2006. 